\title{
A Fuzzy Qualitative Approach for Scene Classification
}

\author{
Chern Hong Lim and Chee Seng Chan \\ Centre of Image and Signal Processing \\ Faculty of Computer Science and Information Technology \\ University of Malaya, 50603 Kuala Lumpur, Malaysia \\ Email: ch_lim@siswa.um.edu.my; cs.chan@um.edu.my
}

\begin{abstract}
Scene classification has been studied extensively in the recent past. Most of the state-of-the-art solutions assumed that scene classes are mutually exclusive. However, this is not true as a scene image may belongs to multiple classes and different people are tend to respond inconsistently even given a same scene image. In this paper, we propose a fuzzy qualitative approach to address this problem. That is, we first adopted the fuzzy quantity space to model the training data. Secondly, we present a novel weight function, $w$ to train a fuzzy qualitative scene model in the fuzzy qualitative states. Finally, we introduce fuzzy qualitative partition to perform the scene classification. Empirical results using a standard dataset and a comparison with K-nearest neighbour has shown the effectiveness and robustness of the proposed method.
\end{abstract}

\section{INTRODUCTION}

Scene classification is an important problem for computer vision, and has been studied extensively in the recent past. It is not an easy task owing to their variability, ambiguity and the wide range of illumination and scale conditions that may apply. Low level features such as colour, texture and shape features have been widely used in combination with supervised learning methods such as support vector machines (SVM), Knearest neighbour $(\mathrm{KNN})$, linear discriminant functions etc. to classify images into several classes (coast, indoor, city, mountain etc.).

Oliva and Torralba [1] proposed a set of perceptual dimensions (naturalness, openness, roughness, expansion, ruggedness) that represent the dominant spatial structure of a scene the spatial envelope as scene representation. Then, a SVM classifier with Gaussian kernel is employed to classify the scene classes. Fei-Fei and Perona [2] proposed the Bayesian hierarchical model extended from latent dirichlet allocation (LDA) to learn natural scene categories. Bosch et al. [3] inspired from this work proposed probabilistic latent semantic analysis (pLSA) incorporate with KNN for scene classification. Vogel and Schiele [4], [5] used the occurring frequency of different concepts (water, rock, etc.) in an image as the intermediate features for scene image classification. The twostage system makes use of an intermediary semantic level of block classification (concept level) to do retrieval based on the occurrence of such concepts in an image. Graphical models were used by Kumar et al. [6] to detect and localize man made structures in a scene, doing in this way scene segmentation and classification.

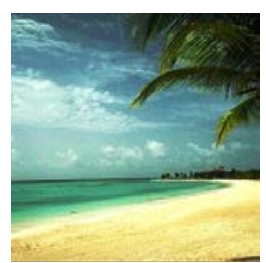

(a) Coast

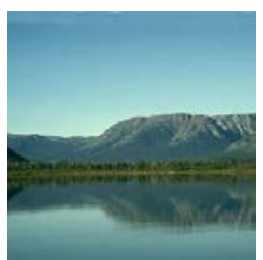

(b) ?

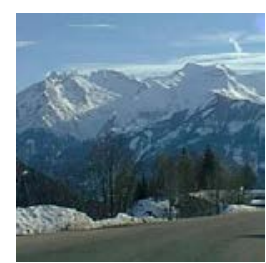

(c) Mountain
Fig. 1. Example of ambiguous scene between coast and mountain

Although significant results have been achieved, classification errors often occur when the classes overlap in the selected feature space. This problem occurred as most of the state-ofthe-art approaches assumed that scene classes are mutually exclusive. That is most systems are exemplar-based, learning patterns from a training set and search for the images "similar" to it, where similarity is often defined only by low level features.

In this paper, we introduce a fuzzy qualitative approach to address this problem. Our approach adopted the fuzzy quantity space [7] and propose a novel weight function, $w$ to model and train a scene model. Then, we introduce the fuzzy qualitative partition to perform classification. The advantages are twofolds. First of all, our approach is able to model the not mutually exclusive data. In scene classification domain, an image may belongs to multiple classes. For instance, it is unclear that if in Fig. 1(b) is a beach scene or a mountain scene. Different people are likely to respond inconsistently in providing the presence or absent of the local features for this image. Secondly, our classification result is not binary instead it is a ranking system to classify each scene image as a combination of different classes. The rank is achieved via the proposed weight function which calculate the occurance of training data of a particular class in the fuzzy qualitative states (FQstate).

This rest of the paper is organized as follows. Section II reviews the past work related to scene classification. Section III revisits the fuzzy quantity space. Section IV presents our proposed method - the fuzzy qualitative approach for scene classification. Section V presents the experiment results using a standard dataset, and we conclude with suggestions of future work in Section VI. 


\section{RELATED WORK}

Scene classification differs from the conventional object detection/classification, to the extent that a scene is composed of several entities often organized in an unpredictable layout [8]. Early efforts at scene classification targeted binary problems, such as distinguishing indoor from outdoor scenes etc. However, in scene classification tasks, it is likely that some scene belongs to multiple classes.

There are some related work that dealt with this issues. Vogel and Schiele [4], [5] used the occurring frequency of different concepts (water, rock, etc.) in an image as the intermediate features for scene image classification. Recently, Parikh and Grauman [9] propose a relative attribute which making the computer to mimic how human classify an object or an action based on the relative attributes.

In what constitutes the closer work to ours, Boutell et al [10] proposed a "cross training" on multi-label approach for scene modelling. Then, a SVM with Gaussian kernel is employed to perform the classification tasks. In contrast to this, our proposed method avoided this hand annotating images during training a scene model as it is tedious and expensive. Also, expert defined classess are somewhat arbitrary and possibly sub-optimal. Our approach train a model directly from the training data in fuzzy qualitative quantity space and our results are defined in a ranking system. That is for example, in Fig. 2, we can notice that Fig. 1(b) actually holds the characteristic of "coast" and "mountain" scene class as it has the water (characteristic of coast scene) and hills (characteristic of mountain scene). Therefore in our classification stage, we propose to represent this relationship as a set of possible scene classes with our novel weight function, $w$.

Fuzzy qualitative reasoning (FQR) is a form of approximate reasoning that can be defined loosely as the fusion of fuzzy reasoning $(\mathrm{FR})$ with qualitative reasoning $(\mathrm{QR})$. Both these research areas have as one of their goals the construction of computational reasoning tools that can predict and explain the behaviour of, often dynamic, systems whose analytic relations are incompletely specified. Whereas pure FR utilizes black box models, QR utilizes explicit structural models. And whereas pure QR operates with symbolic 'quantities', FR explicitly reasons with fuzzy intervals of varying precision that are supported directly by the real number line.

The history of FQR development can be broadly represented by the tools, techniques and method developed to solve real world problems areas as diverse as robotics [11], [12], computer vision [13], process engineering and biology. Perhaps the earliest approach tried was that of Dubois and Prade [14] who combined fuzzy reasoning with Forbus' Qualitative Process Engine. This was closely followed by the work of Shen and Leitch [7] who took a similar general approach but used qualitative simulation (QSIM) as the template for development. QSIM was developed by Kuipers [15], and is a constraint based QR package utilising qualitative differential equations (QDEs) - which are abstractions of ordinary differential equations - to specify the constraints. FuSim [7],

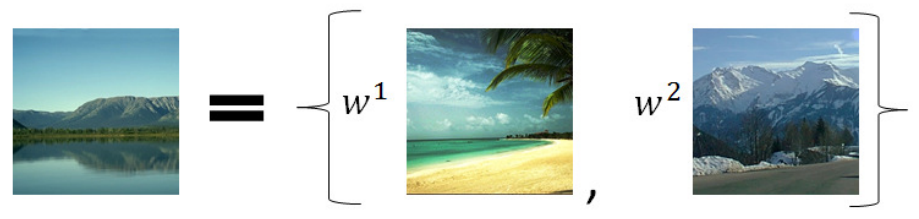

Fig. 2. Examples of relationship of ambiguous class between discrete classes

represents the values of variables as parameterised four-tuple fuzzy numbers which constitute the fuzzy quantity spaces, in contrast to the symbolic values utilised in QSIM. This allows the model to be analysed more precisely over time whilst retaining the essential features of $\mathrm{QR}$. For a detailed review of the recent advances in FQR, please refer to [16].

\section{FuZZY QuANTITy SPACE REVISITS}

A fuzzy quantity space is generated by a finite discrimination of the underlying range of each variable of a system being modelled. The fuzzy quantity space will have the desirable properties of finiteness and coverage, as long as the system contains a finite number of variables. Granularity in the fuzzy quantity space is obtained by the arbitrariness of the discrimination of the numeric ranges of system variables that are assumed to be of interest. Hence, a subset of a numeric range can be translated to one qualitative value according to what is needed in a particular modelling process, such that the extensions of a single qualitative intention may be rather different. The adoption of fuzzy subsets has a direct distinct advantage over the traditional crisp representations when considering granularity.

In fact, if one intends to describe the qualitative values of system variables only in terms of the crisp subsets of the underlying real range of the variables, the mapping from the real range to a quantity space will result in the search for the limits of the real numbers served as the boundaries between (dis-jointly) adjacent qualitative values within the quantity space. This usually incurs severe difficulties in determining these limits [7]. The fuzzy representation of qualitative values is more general than ordinary (crisp) interval representations, since it can represent not only the information stated by a welldetermined real interval but also the knowledge embedded in the soft boundaries of the interval. Thus, fuzzy quantity space removes, or largely weakens (if not completely resolving), the boundary interpretation problem, achieved through the description of a gradual rather than an abrupt change in the degree of membership of which a physical quantity is mapped onto a particular qualitative value. It is, therefore, closer to the common sense intuition of the description of a qualitative value.

This definition on a fuzzy quantity space is given in a general form such that the operations performed within such a quantity space, consisting of normal and convex fuzzy numbers with arbitrary forms of distribution. As a matter of fact, operations on fuzzy qualitative values are based upon the 
extension principle outlined in [7]. This principle is invoked every time an arithmetic operation is performed and requires expensive calculation. Also, the computational implementation of the calculation with arbitrary membership distributions of fuzzy numbers can only be done in a discrete domain obtained by sampling the original continuous distribution. The use of the extension principle with sampled membership distributions generates a considerable increase in the discrete samples of the result, and furthermore, only some of the resulting samples are correct. Fortunately, computationally more efficient ways to characterise fuzzy numbers have been developed. This utilises a parametric approximation of the membership function where the membership distribution of a normal convex fuzzy number is approximated by the 4-tuples, [a, b, $\alpha, \beta]$.

An example of which in FuSim [7] is shown in Figure 3, and defined as,

$$
\mu_{A}(x)=\left\{\begin{array}{cc}
0 & x<a-\tau \\
\tau^{-1}(x-a+\tau) & x \in[a-\tau, a] \\
1 & x \in[a, b] \\
\beta^{-1}(b+\beta-x) & x \in[b, b+\beta] \\
0 & x>b+\beta
\end{array}\right.
$$

The arithmetic operations on these fuzzy numbers are well developed and for the preceding reasons, [7] adopt such a representation to form the fuzzy quantity space in this work. A fuzzy quantity space formed in this way makes it possible to build a bridge between 'sets' and 'value' because representation allows a real number, a real interval, a fuzzy number, and a fuzzy interval to be uniformly described. Thus, the qualitative category representation and the ordinal representation can be combined in a natural way. For example, the real number 4 can be denoted by a real interval $[4,4]$, which in turn, can be represented by a 4-tuples fuzzy number [4, 4, 0, 0], whilst this fuzzy number is a special fuzzy subset of the real line.

Similarly, the real interval $[3.8,4]$ can be represented by the fuzzy description $[3.8,4,0,0]$, and the strict fuzzy number 'approximately 4' may be expressed by [4, 4, 3, 3]. In this way, when there does exist a precise qualitatively distinct landmark value, this value can also be represented in the form of a 4tuples number. Furthermore, even if the landmarks are only partially known, say, in terms of the lower and upper (exact) boundaries of the range within which a landmark value falls, such knowledge can still be encoded by the 4-tuples version of a real interval as shown above.

In this paper, our fuzzy quantity space $F Q S$ is denoted as,

$$
F Q S=\left[F Q S^{x}, F Q S^{y}\right]
$$

where

$$
\begin{aligned}
& F Q S^{x}=\left[Q S T_{x}^{1}, Q S T_{x}^{2}, \cdots, Q S T_{x}^{n}\right] \\
& F Q S^{y}=\left[Q S T_{y}^{1}, Q S T_{y}^{2}, \cdots, Q S T_{y}^{m}\right]
\end{aligned}
$$

$Q S T_{x}$ and $Q S T_{y}$ are the fuzzy qualitative states (FQstates) along the $x$-axis and $y$-axis, respectively. $n$ is the total number

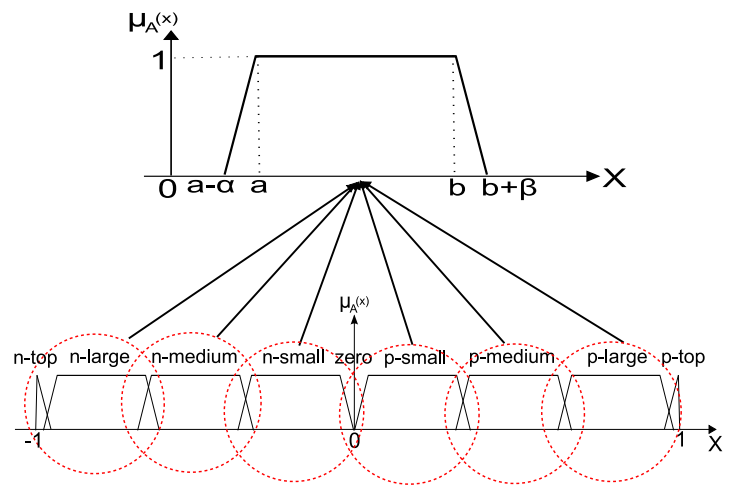

Fig. 3. 4-tuples fuzzy quantity space

of $Q S T_{x}$ in $x$-axis, and $m$ is the total number of $Q S T_{y}$ in $y$-axis.

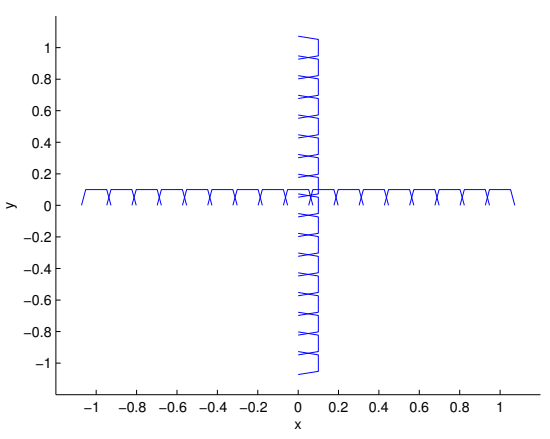

Fig. 4. An example of the fuzzy quantity space with $n=17$ and $m=17$ 5.

The FQstate is represented by a 4-tuples number as in Eq.

$$
S T=[a, b, \alpha, \beta]
$$

where $a$ and $b$ are the dominant values and, $\alpha$ and $\beta$ are the tolerance values of the FQstate respectively. A 2D-FQstate can be composed from two FQstates with each at different axis, Fig. 5.

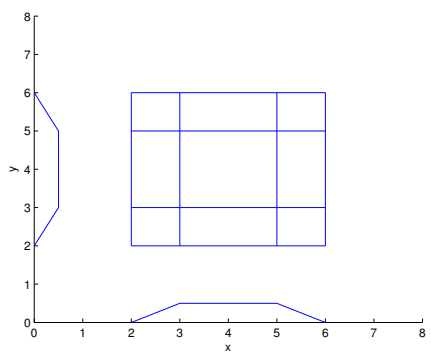

Fig. 5. 2D-FQstate

\section{Fuzzy Qualitative Scene Classification}

In general, scene classification consists of 2 stages, that is a) modelling and b) classification. The ability to analyse and 
classify accurately and rapidly the scene is which we find ourselves is highly useful in everyday life. In this paper, we proposed a fuzzy qualitative approach to scene classification.

\section{A. Modelling}

In order to build a fuzzy qualitative scene model (FQSM) from training data, $T$, we firstly normalized the training data within the range of [-1 1$]$. Secondly, this normalized training data, $T^{\prime}$ are mapped into the FQS in order to build FQSM $\left(T^{\prime} \mapsto F Q S\right)$. Let assume we have a total of $n \times m$ 2DFQstates in the FQS. FQSM can be represented as:

$$
F Q S M=\left\{Q S T^{(1,1)}, Q S T^{(1,3)}, \cdots, Q S T^{(2,2)}, \cdots, Q S T^{(i, j)}\right\}
$$

where $i \leq n$ and $j \leq m$. In training the FQSM, we propose a novel weight function, $w$

$$
w^{k}=\frac{N_{k}}{\sum_{k=1}^{K} N_{k}}
$$

where $N_{k}$ is the occurance number of $T^{\prime}$ of a particular class, $k$ in a 2D-FQstate. Therefore, in each 2D-FQstates in the FQSM, there is a weight that correspond to each scene class as to Eq. 8 .

$$
Q S T^{(i, j)}=\left\{w^{1}, w^{2}, \cdots, w^{K}\right\}
$$

where $K$ is the total number of classes that map into the FQS. For example, if $K=3$, each 2D-FQstates in the FQSM will be represented as $Q S T^{(i, j)}=\left\{w^{1}, w^{2}, w^{3}\right\}$ and $\sum w=1$. The advantage of this approach is we do not assume scene class are mutually exclusiove but a combination of them.

As an example, Fig. 6 show a FQSM with $K=3$ in the FQS and the algorithm for fuzzy qualitative modelling is shown in Algorithm 1.

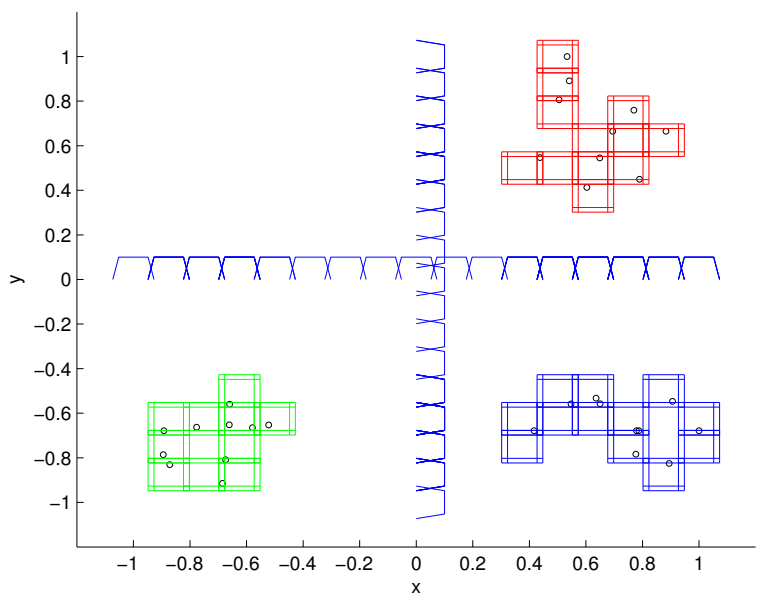

Fig. 6. An example of FQSM with $K=3$

\section{Algorithm 1 FUZZY QUALITATIVE SCENE CLASSIFICATION} - MODELLING STAGE

Require: A training dataset

Ensure: Fuzzy qualitative space (FQS) is built

Ensure: The training data are normalized

Map the normalized training data to the FQS

Calculate the weight, $w$ of each class in each 2D-FQstate as Eq. 7

return $\mathrm{FQSM}$

\section{B. Classification}

For classification stage, let us denote $d=\left[d_{x}, d_{y}\right]$ as a set normalized testing data. In order to choose the most likely 2D-FQstate that $d$ belongs to, we introduce fuzzy qualitative partition (FQP). FQP consists of nine partitions derived from the 2D-FQstate where each partition has different degree of membership, $\mu$ (Fig. 7). The notation of each partition of the 2D-FQstate is represented in Table I.

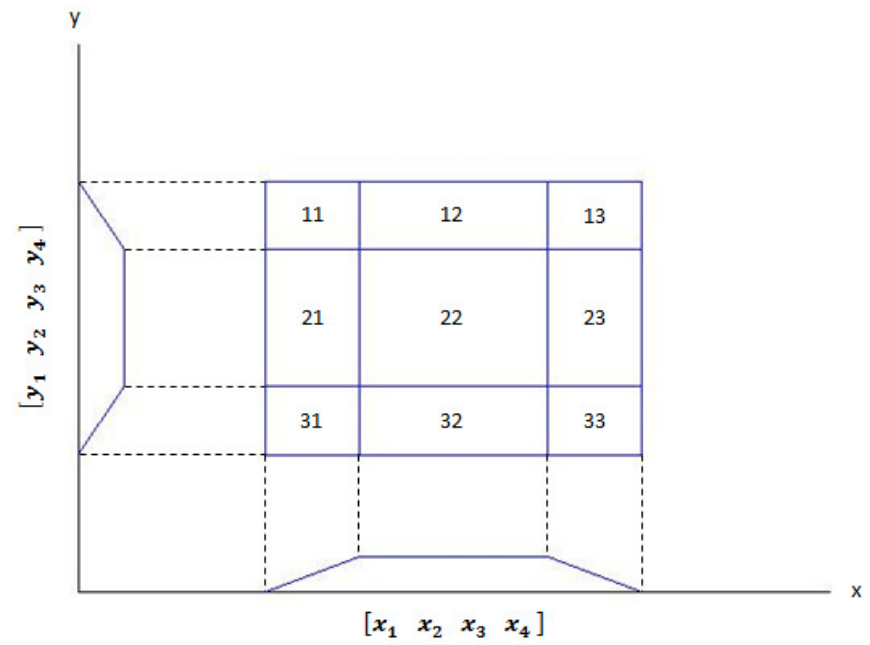

Fig. 7. Fuzzy Qualitative Partition

TABLE I

NOTATION OF FQP

\begin{tabular}{|l|c|c|c|}
\hline & $x_{1} \leqslant d_{x} \leqslant x_{2}$ & $x_{2} \leqslant d_{x} \leqslant x_{3}$ & $x_{3} \leqslant d_{x} \leqslant x_{4}$ \\
\hline$y_{3} \leqslant d_{y} \leqslant y_{4}$ & $P^{(11)}$ & $P^{(12)}$ & $P^{(13)}$ \\
\hline$y_{2} \leqslant d_{y} \leqslant y_{3}$ & $P^{(21)}$ & $P^{(22)}$ & $P^{(23)}$ \\
\hline$y_{1} \leqslant d_{y} \leqslant y_{2}$ & $P^{(31)}$ & $P^{(32)}$ & $P^{(33)}$ \\
\hline
\end{tabular}

For example, the FQP gives the intuition of: $P^{(22)}$ denote the FQP where both the degree of membership of the $x$ and $y$ axis are $1 . P^{(12)}, P^{(21)}, P^{(32)}, P^{(23)}$ denote the $\mathrm{FQP}$ in which either degree of membership of the $x$ or $y$ axis is 1. $P^{(11)}, P^{(13)}, P^{(31)}, P^{(33)}$ denote the FQP in which neither degree of membership of the $x$ or $y$ axis is 1 . 
TABLE II

$\mu$ CALCULATION IN FQP

\begin{tabular}{|l|c|c|c|}
\hline & $x_{1} \leqslant d_{x} \leqslant x_{2}$ & $x_{2} \leqslant d_{x} \leqslant x_{3}$ & $x_{3} \leqslant d_{x} \leqslant x_{4}$ \\
\hline$y_{3} \leqslant d_{y} \leqslant y_{4}$ & $\frac{d_{x}-x_{1}}{x_{2}-x_{1}} \times \frac{y_{4}-d_{y}}{y_{4}-y_{3}}$ & $1 \times \frac{y_{4}-d_{y}}{y_{4}-y_{3}}$ & $\frac{x_{4}-z_{x}}{x_{4}-x_{3}} \times \frac{y_{4}-d_{y}}{y_{4}-y_{3}}$ \\
\hline$y_{2} \leqslant d_{y} \leqslant y_{3}$ & $\frac{d_{x}-x_{1}}{x_{2}-x_{1}} \times 1$ & $1 \times 1$ & $\frac{x_{4}-d_{x}}{x_{4}-x_{3}} \times 1$ \\
\hline$y_{1} \leqslant d_{y} \leqslant y_{2}$ & $\frac{d_{x}-x_{1}}{x_{2}-x_{1}} \times \frac{d_{y}-y_{1}}{y_{2}-y_{1}}$ & $1 \times \frac{d_{y}-y_{1}}{y_{2}-y_{1}}$ & $\frac{x_{4}-d_{x}}{x_{4}-x_{3}} \times \frac{d_{y}-y_{1}}{y_{2}-y_{1}}$ \\
\hline
\end{tabular}

However, there are sometimes $d$ will fall into more than one 2D-FQstates, we denote it as $l>1$ where $l=\{1,2,4\}$. This will happen when $d$ falls into the FQP as below:

- $d$ belongs to two 2D-FQstates, $l=2$ when it falls into $P^{(12)}, P^{(21)}, P^{(32)}$, and $P^{(23)}$.

- $d$ belongs to four 2D-FQstates, $l=4$ when it falls into $P^{(11)}, P^{(13)}, P^{(31)}$, and $P^{(33)}$.

In order to choose the most possible 2D-FQstate that $d$ belongs to, a degree of membership for each 2D-FQstate corresponds to $d, \mu_{d}$ is calculated based on Table II. From the calculation of $\mu_{d}$, the 2D-FQstate that holds the highest degree of membership toward $d, Q S T^{C}$ as Eq. 9 will be selected.

$$
Q S T^{C}=\max \left\{\mu_{d}^{Q S T^{1}}, \mu_{d}^{Q S T^{2}}, \cdots, \mu_{d}^{Q S T^{l}}\right\}
$$

Then, within this $Q S T^{C}$, we will use the trained weight function, $w$ to perform scene classification.

$$
Q S T^{C}=\left\{w^{1}, w^{2}, w^{3}\right\}
$$

where Eq. 10 shows us that $d$ holding the degree of $w^{1}$ belongs to Class $=1, w^{2}$ belongs to Class $=2$, and $w^{3}$ belongs to Class $=3$. This is one of the advantage of our proposed approach that we are not going to ignore any possibility that $d$ could belongs to any class compared to previous solutions that assume scene classes are mutually exclusive where it will produce only one classification result at the final stage.

The algorithm for fuzzy qualitative scene classification is shown in Algorithm 2.

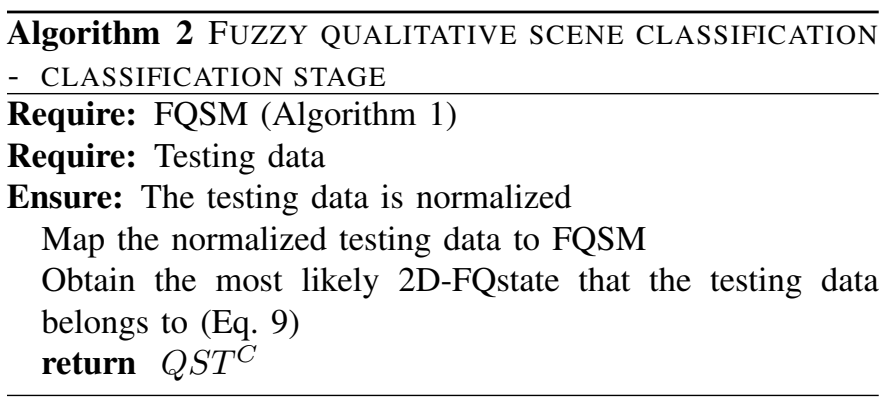

\section{EXPERIMENTAL RESULTS}

In order to test the effectiveness and robustness of our proposed framework, we tested the proposed method using the Outdoor Scene Recognition (OSR) Dataset [1]. A total of four classes of the scenes are used throughout the experiments which are 'Insidecity', 'Coast', 'Opencountry', and 'Forest'. The examples of those scenes are shown in Fig. 8. These four classes of the scenes are chosen in our experiments because each of them have their own unique characteristics correspond to the degree of 'Openness' and degree of 'Naturalness'. As for example, coast scene have high value of Openness while forest scene have low value of Openness. Fig. 9 shows the original distribution of the four classes of scene correspond to the degree of the attributes. The attributes we used (degree of 'Openness' and degree of 'Naturalness') are introduced in [1] and called the spatial envelope properties. The score of each image for each attributes in our experiments are computed by [9] and the source are available for public in http://ttic.uchicago.edu/ dparikh/relative.html.

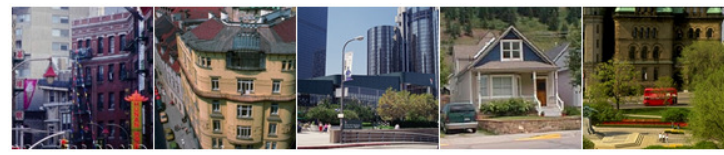

(a) Examples of insidecity scene

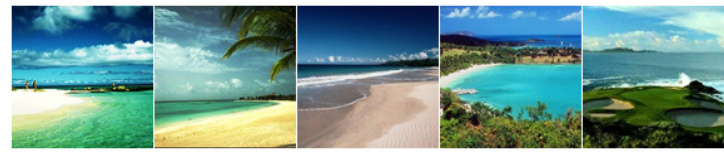

(b) Examples of coast scene

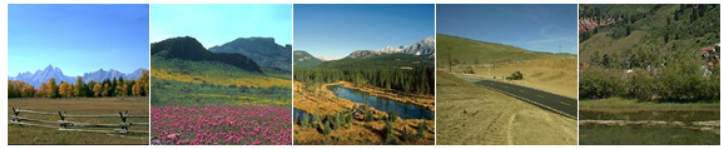

(c) Examples of opencountry scene

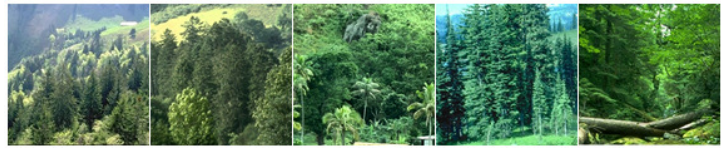

(d) Examples of forest scene

Fig. 8. Examples of the scenes from four classes

\section{A. Scene Classification}

In this experiments, we used 'leave-one-out' method to classify insidecity scenes and opencountry scenes. Fig. 10 illustrates the insidecity scenes and Table III presents the classification results. Similarly, Fig. 12 illustrates the opencountry scenes and Table IV presents the classification results.

From the achieved results (Table III-IV), it shows the effectiveness and robustness of our proposed approach. For example, our proposed method confidently classified both the Fig. 10(a) to 10(b) and Fig. 12(a) toward insidecity and opencountry class respectively with $w=1$. This is because in Fig. 10(a) to 10(b) have low degree of Openness and low 


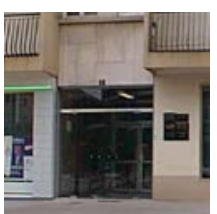

(a)

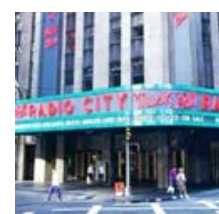

(b)

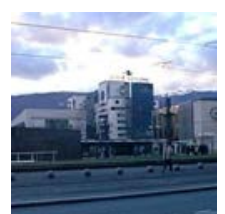

(c)

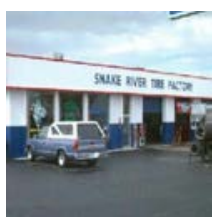

(d)

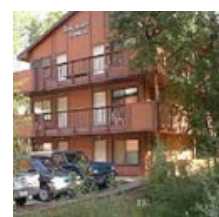

(e)

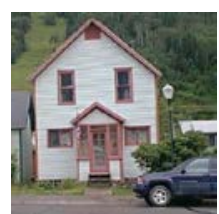

(f)

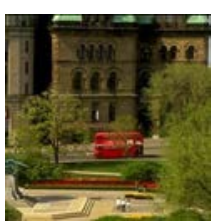

(g)

Fig. 10. Examples of insidecity annotated scenes

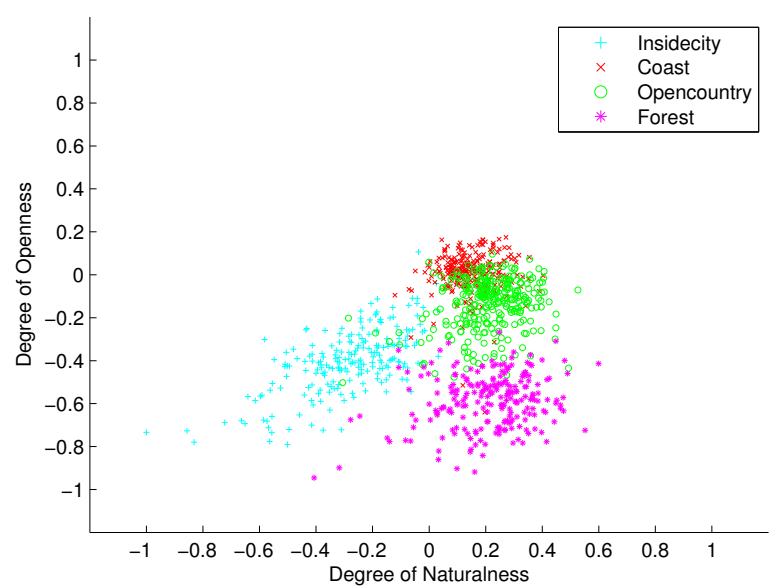

Fig. 9. The distribution of four classes of scenes correspond to the degree of the attributes

TABLE III

FUZZY CLASSIFICATION OUTPUTS FOR INSIDECITY SCENES CLASSIFICATION

\begin{tabular}{|c|c|c|c|c|}
\hline \multirow{2}{*}{ Scene } & \multicolumn{4}{|c|}{ Weight, $w$} \\
\cline { 2 - 5 } & Insidecity & Coast & Opencountry & Forest \\
\hline $10(\mathrm{a})$ & 1 & 0 & 0 & 0 \\
\hline $10(\mathrm{~b})$ & 1 & 0 & 0 & 0 \\
\hline $10(\mathrm{c})$ & 0.7273 & 0.2727 & 0 & 0 \\
\hline $10(\mathrm{~d})$ & 0.7273 & 0.2727 & 0 & 0 \\
\hline $10(\mathrm{e})$ & 0.1250 & 0 & 0.1250 & 0.7500 \\
\hline $10(\mathrm{f})$ & 0.8235 & 0 & 0 & 0.1765 \\
\hline $10(\mathrm{~g})$ & 0.8235 & 0 & 0 & 0.1765 \\
\hline
\end{tabular}

degree of Naturalness which are the characteristics of insidecity scenes. Then, Fig. 10(c) to 10(d) hold the combination toward insidecity class and also coast class because they have the characteristics of coast scenes which are high degree of Openness and high degree of Naturalness. Fig. 12(b) to 12(d) show the combination of coast class and opencountry class, respesively. In the meantime, Fig. 10(e) to $10(\mathrm{~g})$ hold the degree of belonging toward insidecity class and also forest class because of the low degree of Openness and the high degree of Naturalness is detected from those scenes and these are the characteristics of forest scenes. However, they do not hold the degree of belonging to the coast class as their degree of Openness is not the threshold of coast scenes.

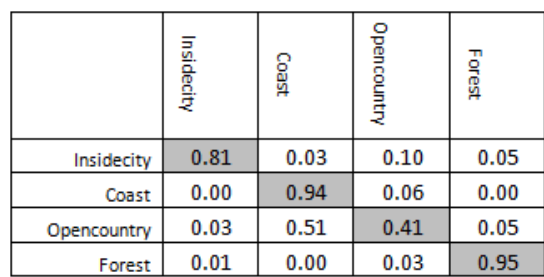

(a) $n=9$ and $m=9$

\begin{tabular}{|c|c|c|c|c|}
\hline & $\begin{array}{l}\frac{\overline{\bar{v}}}{\overline{\frac{\omega}{0}}} \\
\frac{\mathrm{D}}{\mathrm{v}}\end{array}$ & 总 & 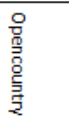 & 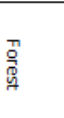 \\
\hline Insidecity & 0.89 & 0.02 & 0.05 & 0.02 \\
\hline Coast & 0.01 & 0.76 & 0.21 & 0.01 \\
\hline Opencountry & 0.04 & 0.17 & 0.73 & 0.05 \\
\hline Forest & 0.02 & 0.00 & 0.03 & 0.92 \\
\hline
\end{tabular}

(b) $n=17$ and $m=17$

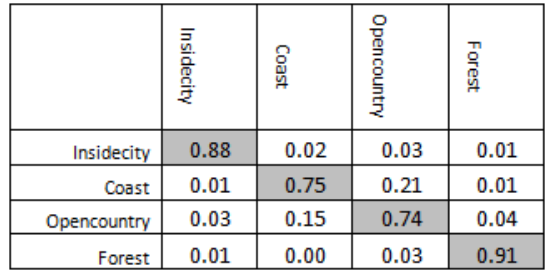

(c) $n=25$ and $m=25$

\begin{tabular}{|c|c|c|c|c|}
\hline & 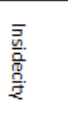 & $\stackrel{8}{\ddot{\sharp}}$ & 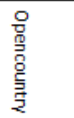 & 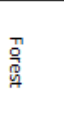 \\
\hline Insidecity & 0.83 & 0.01 & 0.03 & 0.01 \\
\hline Coast & 0.01 & 0.77 & 0.18 & 0.01 \\
\hline Opencountry & 0.02 & 0.19 & 0.72 & 0.04 \\
\hline Forest & 0.01 & 0.00 & 0.03 & 0.87 \\
\hline
\end{tabular}

(d) $n=33$ and $m=33$

Fig. 11. Confusion matrixes of crisp classification results for different $q x$ and $q y$ values

\section{B. System accuracy}

In this experiment, we would like to test the accuracy of our proposed approach in classify scene. The groundtruth is of this is provided by the (OSR) Dataset [1]. The results are based on the average outcome from 20 iterations with $70 \%$ of training data and $30 \%$ of testing data. Fig. 13 shows the examples of the FQSM with different resolutions. For better understanding on how a FQSM is built, Fig. 14 visualize the examples of the FQSM for each class of the scenes with $n=17$ and $m=17$.

Confusion matrixes of this experiment are shown in Fig. 11. 


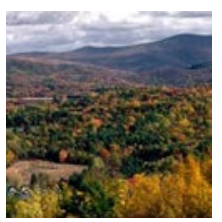

(a)

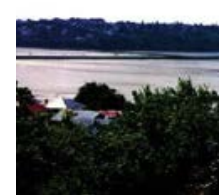

(b)

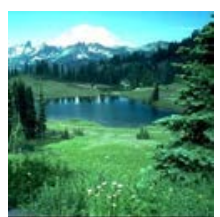

(c)

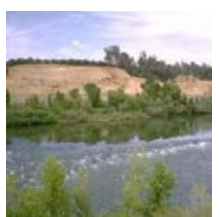

(d)

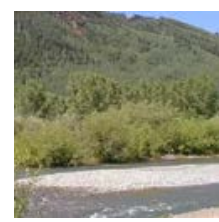

(e)

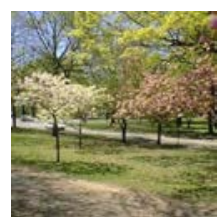

(f)

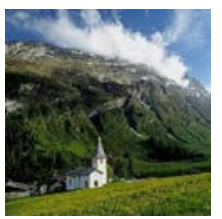

(g)

Fig. 12. Examples of opencountry annotated scenes

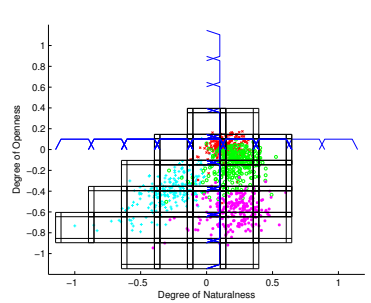

(a) $n=9$ and $m=9$

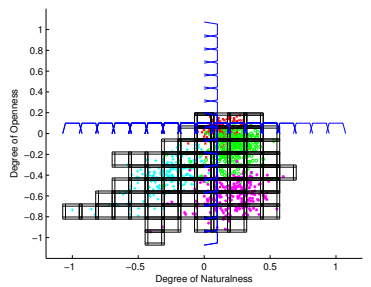

(b) $n=17$ and $m=17$

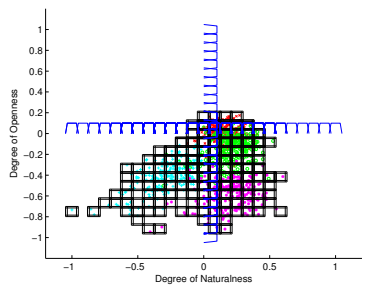

(c) $n=25$ and $m=25$

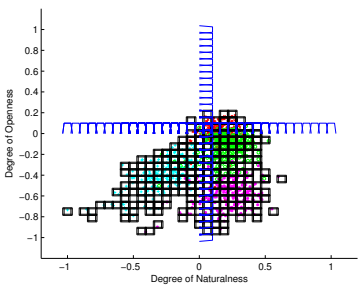

(d) $n=33$ and $m=33$

Fig. 13. Examples of FQSM with different resolutions

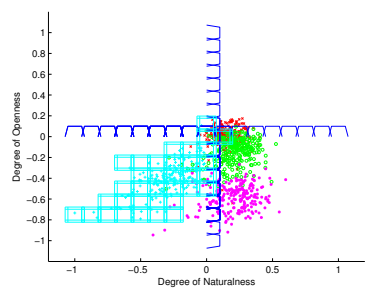

(a) Insidecity

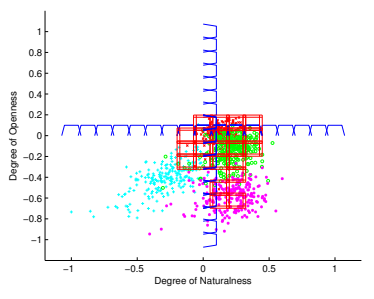

(b) Coast

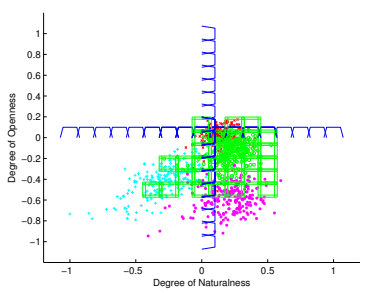

(c) Opencountry

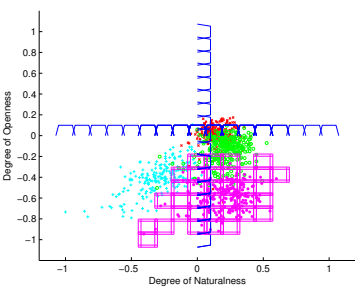

(d) Forest

Fig. 14. Examples of FQSM for each class scene with $n=17$ and $m=17$

TABLE IV

FUZZY CLASSIFICATION OUTPUTS FOR OPENCOUNTRY SCENES CLASSIFICATION

\begin{tabular}{|c|c|c|c|c|}
\hline \multirow{2}{*}{ Scene } & \multicolumn{4}{|c|}{ Weight, $w$} \\
\cline { 2 - 5 } & Insidecity & Coast & Opencountry & Forest \\
\hline $12(\mathrm{a})$ & 0 & 0 & 1 & 0 \\
\hline $12(\mathrm{~b})$ & 0 & 0.1111 & 0.8889 & 0 \\
\hline $12(\mathrm{c})$ & 0 & 0.0435 & 0.9130 & 0.0435 \\
\hline $12(\mathrm{~d})$ & 0 & 0.3387 & 0.6613 & 0 \\
\hline $12(\mathrm{e})$ & 0 & 0 & 0.6471 & 0.3529 \\
\hline $12(\mathrm{f})$ & 0 & 0 & 0.0233 & 0.9767 \\
\hline $12(\mathrm{~g})$ & 0 & 0.0435 & 0.3913 & 0.5652 \\
\hline
\end{tabular}

From the results, we observed that in general our proposed approach has a stable accuracy eventhough using different resolutions. The average accuracy (\%) is $80.5 \pm 2.5$ and we found that $n=17$ and $m=17$ holds the best accuracy. The poorest result is when $n=9$ and $m=9$ where our proposed approach confuse between "Opencountry" and "Coast". This is because these two scenes are quite similar to each other and thus having a lot of cross over data especially most opencountry scenes are crossing towards coast scenes.

\section{Comparison with KNN}

Secondly, we evaluated the performance of our proposed method using different training data resolution $(70 \%, 50 \%$, and $30 \%)$ where the remaining data $(100 \%$ - \% of training data) will be the testing data and a comparison with KNN. We obtain the crisp classification result for a particular testing data by selecting the scene class which has the highest weight, $w$ from the respective 2D-FQstates that the testing data falls in. From Table V, first of all it shows that our results are inline with $\mathrm{KNN}$ and this proves that our approach do has the ability to perform crisp classification as well. However, our approach is better than KNN in terms we do not assume scene classes are mutually exclusive. We allow a scene class to belong to multiple classes. Secondly, our proposed approach does not effect by the size of training data. Althought we only use $30 \%$ of the OSR datset as training data, in comparison to use $70 \%$ of the OSR dataset as training data, the accuracy only differ by $\pm 4 \%$.

\section{CONCLUDING REMARKS}

In this paper, we show the implementation of $\mathrm{FQS}$ and the usage of it in natural scenes classification. The experiments show positive results in term of crisp classification and fuzzy classification results. However, there are more research to be 
TABLE V

COMPARISON WITH KNN BASED ON DIFFERENT \% OF TRAINING DATA

\begin{tabular}{|c|c|c|c|c|c|c|c|c|}
\hline \multirow{2}{*}{$\begin{array}{c}\text { Training data } \\
(\%)\end{array}$} & \multicolumn{4}{|c|}{ Accuracy for FQS (\%) } & \multicolumn{4}{|c|}{ Accuracy for KNN (\%) } \\
\hline & Insidecity & Coast & Opencountry & Forest & Insidecity & Coast & Opencountry & Forest \\
\hline 70 & 0.89 & 0.76 & 0.73 & 0.92 & 0.89 & 0.70 & 0.65 & 0.92 \\
\hline 50 & 0.88 & 0.77 & 0.69 & 0.91 & 0.91 & 0.70 & 0.67 & 0.91 \\
\hline 30 & 0.87 & 0.77 & 0.69 & 0.89 & 0.89 & 0.70 & 0.70 & 0.91 \\
\hline
\end{tabular}

done to fine tune the proposed framework. In the future, we intent to solve the problem on choosing the optimal $n$ and $m$ values for better classification result. Besides that, another limitation of our proposed framework which we wish to solve is the unidentified data problem when the new entry data do not fall into any of the 2D-FQstates in the FQSM during classification stage. Furthermore, we hope to enhance our proposed framework to be able to support multivariable (where we used just two variables in our implementation) and also dynamic fuzzy sets in each FQstate which the fuzzy sets values may obtain from human experts or some optimization algorithms. Finally, we hope to obtain the relative relationship information such as 'coast is more open than forest' in order to increase the resolution of the classification result.

\section{ACKNOWLEDGMENT}

This work is supported in part by the UM-HIR (UM.C/625/1/HIR/037, J0000073579); UMRG (RG110$11 \mathrm{AET}$ ) and the UM-BrightSpark Programme.

\section{REFERENCES}

[1] A. Oliva and A. Torralba, "Modeling the shape of the scene: A holistic representation of the spatial envelope," International Journal of Computer Vision, vol. 42, no. 3, pp. 145-175, 2001.
[2] L. Fei-Fei and P. Perona, "A bayesian hierarchical model for learning natural scene categories," 2005 IEEE Computer Society Conference on Computer Vision and Pattern Recognition CVPR05, vol. 2, no. 15, pp. 524-531, 2005.

[3] A. Bosch, A. Zisserman, and X. Mu, "Scene classification via plsa," Analysis, vol. 3954, pp. 517-530, 2006.

[4] J. Vogel and B. Schiele, "Natural scene retrieval based on a semantic modeling step," Image and Video Retrieval, vol. 3115, pp. 1950-1950, 2004.

[5] - "Semantic modeling of natural scenes for content-based image retrieval," International Journal of Computer Vision, vol. 72, no. 2, pp. 133-157, 2007.

[6] S. Kumar and M. Hebert, "Discriminative random fields: A discriminative framework for contextual interaction in classification," in Proceed ings of the Ninth IEEE International Conference on Computer Vision Volume 2, ser. ICCV '03, 2003, pp. 1150-1157.

[7] Q. Shen and R. Leitch, "Fuzzy qualitative simulation," IEEE Transactions On Systems Man And Cybernetics, vol. 23, no. 4, pp. 1038-1061, 1993.

[8] P. Quelhas, F. Monay, J.-M. Odobez, D. Gatica-Perez, T. Tuytelaars, and L. Van Gool, "Modeling scenes with local descriptors and latent aspects," in Proceedings of the Tenth IEEE International Conference on Computer Vision (ICCV'05) Volume 1, 2005, pp. 883-890.

[9] D. Parikh and K. Grauman, "Relative attributes," ICCV, 2011.

[10] M. Boutell, J. Luo, X. Shen, and C. Brown, "Learning multi-label scene classification," Pattern Recognition, vol. 37, no. 9, pp. 1757-1771, 2004.

[11] H. Liu, D. J. Brown, and G. M. Coghill, "Fuzzy qualitative robot kinematics," IEEE Transactions on Fuzzy Systems, vol. 16, no. 3, pp. 808-822, 2008.

[12] H. Liu, G. M. Coghill, and D. P. Barnes, "Fuzzy qualitative trigonometry," International Journal of Approximate Reasoning, vol. 51, no. 1, pp. 71-88, 2009.

[13] C. S. Chan and H. Liu, "Fuzzy qualitative human motion analysis," IEEE Transactions on Fuzzy Systems, vol. 17, no. 4, pp. 851-862, 2009.

[14] D. Dubois and H. Prade, "Fuzzy arithmetic in qualitative reasoning," Lecture Notes in Control and Information Sciences, vol. 212, pp. 457467, 1989.

[15] B. Kuipers, "Qualitative reasoning," MIT Press, 1994.

[16] C. S. Chan, G. M. Coghill, and H. Liu, "Recent advances in fuzzy qualitative reasoning," International Journal of Uncertainty, Fuzziness and Knowledge-Based Systems, vol. 19, no. 3, pp. 417-422, 2011. 